MSC 45K05, 47D06, 34K30

DOI: $10.14529 / \mathrm{mmp} 170104$

\title{
REGULARITY RESULTS AND SOLUTION SEMIGROUPS FOR RETARDED FUNCTIONAL DIFFERENTIAL EQUATIONS
}

A. Favini, Department of Mathematics, University of Bologna, Bologna, Italy, favini@dm.unibo.it,

H. Tanabe, Takarazuka, Japan, bacbx403@jttk.zaq.ne.jp

We show that the solutions of the retarded functional differential equations in a Banach space, whose existence and uniqueness are established in paper of A. Favini and H. Tanabe, have some further regularity properties if the initial data and the inhomogeneous term satisfy some smootheness assumptions. Some results on the solution semigroups analogous to the one of G. Di Blasio, K. Kunisch and E. Sinestrari and to the one of E. Sinestrari are also obtained.

Keywords: retarded functional differential equation; regularity of solutions; analytic semigroup; solution semigroup; $C_{0}$-semigroup; infinitesimal generator.

\section{Introduction}

We consider the following retarded functional differential equation in a complex Banach space $X$ :

$$
\left\{\begin{array}{l}
\frac{d}{d t} u(t)=A u(t)+A_{1} u(t-r)+\int_{-r}^{0} a(s) A_{2} u(t+s) d s+f(t), 0 \leq t \leq T, \\
u(0)=\varphi_{0}, \quad u(s)=\varphi_{1}(s) \text { a.e. } s \in(-r, 0) .
\end{array}\right.
$$

We assume that $A$ is a densely defined closed linear operator which generates an analytic semigroup $e^{t A}, t \geq 0$, in $X$. Suppose $0 \in \rho(A)$ for simplicity. $A_{1}$ and $A_{2}$ are closed linear operators in $X$ such that $D\left(A_{1}\right) \supset D(A), D\left(A_{2}\right) \supset D(A)$, and $a$ is a complex valued function defined in the interval $[-r, 0]$ such that $a \in L^{1}(-r, 0 ; \mathbb{C})$.

The following theorems which are improvements of the results by G. Di Blasio and A. Lorenzi [1] are established in A. Favini and H. Tanabe [2]:

Theorem A Suppose $0<\theta<1 / p$. If the following assumption is satisfied:

(I) $\varphi_{0} \in(X, D(A))_{\theta+1-1 / p, p}, \quad \varphi_{1} \in W^{\theta, p}(-r, 0 ; D(A)), f \in W^{\theta, p}(0, T ; X)$,

then, there exists a unique solution $u$ of (0.1) satisfying

$$
\begin{aligned}
& u \in W^{\theta, p}(0, T ; D(A)) \cap C\left([0, T] ;(X, D(A))_{\theta+1-1 / p, p}\right), \\
& d u / d t \in W^{\theta, p}(0, T ; X) .
\end{aligned}
$$

Theorem B Suppose $1 / p<\theta<1$. If the following assumption is satisfied:

$$
\left\{\begin{array}{l}
\varphi_{0} \in D(A), \quad \varphi_{1} \in W^{\theta, p}(-r, 0 ; D(A)), \quad \varphi_{1}(0)=\varphi_{0}, \\
f \in W^{\theta, p}(0, T ; X) \cap C\left([0, T] ;(X, D(A))_{\theta-1 / p, p}\right), \\
A \varphi_{0}+A_{1} \varphi_{1}(-r)+\int_{-r}^{0} a(s) A_{2} \varphi_{1}(s) d s \in(X, D(A))_{\theta-1 / p, p},
\end{array}\right.
$$


then, problem (1.1) admits a unique solution u such that

$$
\begin{aligned}
& u \in W^{\theta, p}(0, T ; D(A)), \\
& d u / d t \in W^{\theta, p}(0, T ; X) \cap C\left([0, T] ;(X, D(A))_{\theta-1 / p}\right) .
\end{aligned}
$$

Note that since $A_{2} \varphi_{1} \in C([-r, 0] ; X)$, the integral $\int_{-r}^{0} a(s) A_{2} \varphi_{1}(s) d s$ is well defined.

In this paper we prove further regularity of solutions $u$ when $\varphi_{1}$ and $f$ satisfy more regularity assumptions. Using this result we prove some results on solution semigroups analogous to the one of G. Di Blasio, K. Kunisch and E. Sinestrari [3] when $\theta<1 / p$, and to the one of E. Sinestrari [4] when $\theta>1 / p$. In case $\theta<1 / p$ it is shown that the map $\left(\begin{array}{c}\varphi_{0} \\ \varphi_{1}\end{array}\right) \mapsto\left(\begin{array}{c}u(t) \\ u_{t}\end{array}\right)$, where $u$ is the solution of $(0.1)$ with $f(t) \equiv 0$ and $u_{t}(s)=u(t+s),-r \leq s \leq 0$, is a $C_{0}$-semigroup in $(X, D(A))_{\theta+1-1 / p, p} \times W^{\theta, p}(-r, 0 ; D(A))$, and the characterization of its infinitesimal generater is given. This is nothing but a simple extention of a result of [3] in case where $X$ is a Hilbert space and $\theta=1$, $p=2$. However, in case $\theta>1 / p$ the situation is a little more complicated. This is caused by the following fact. In this case there appears the space $(X, D(A))_{\theta+1-1 / p, p}$ which is a subset of $D(A)$. If $u$ belongs to this space, $A_{i} u, i=1,2$, is defined, but may not belong to $(X, D(A))_{\theta-1 / p, p}$. Therefore we assume the additional condition $A_{i} A^{-1} \in \mathcal{L}\left((X, D(A))_{\theta-1 / p, p}\right), i=1,2$. A comment on this assumption will be given in section 6. Under these hypotheses it will be shown that the map $\varphi_{1} \mapsto u_{t}$ is a $C_{0}$-semigroup in $W^{\theta, p}(-r, 0 ; D(A)) \cap C\left([-r, 0] ;(X, D(A))_{\theta+1-1 / p, p}\right)$ with the characterization of its infinitesimal generater, where again $u$ is the solution of $(0.1)$ with $f(t) \equiv 0$ and $u_{t}(s)=u(t+s),-r \leq s \leq 0$.

For a Banach space $Y$ we use the following norm of $W^{\theta, p}(0, T ; Y)$ :

$$
\begin{aligned}
N_{\theta, p, Y,(0, T)}(u) & =\left(\int_{0}^{T} \int_{0}^{t}\|u(t)-u(s)\|_{Y}^{p}(t-s)^{-1-\theta p} d s d t\right)^{1 / p}, \\
\|u\|_{W^{\theta, p}(0, T ; Y)} & =N_{\theta, p, Y,(0, T)}(u)+T^{-\theta}\|u\|_{L^{p}(0, T ; Y)} .
\end{aligned}
$$

\section{Regularity of Solutions: Case $\theta<1 / p$}

First consider the case $0<\theta<1 / p$. Assume that:

$$
\left\{\begin{array}{l}
\varphi_{1} \in W^{1+\theta, p}(-r, 0 ; D(A)), \quad \varphi_{0}=\varphi_{1}(0)(\in D(A)) \\
f \in W^{1+\theta, p}(0, T ; X) \cap C\left([0, T] ;(X, D(A))_{\theta+1-1 / p, p}\right) \\
A \varphi_{0}+A_{1} \varphi_{1}(-r)+\int_{-r}^{0} a(s) A_{2} \varphi_{1}(s) d s \in(X, D(A))_{\theta+1-1 / p, p} .
\end{array}\right.
$$

Theorem 1. Suppose $0<\theta<1 / p$. Then, under assumption (I-1) the solution of problem (0.1) satisfies

$$
u \in W^{1+\theta, p}(0, T ; D(A)) \cap W^{2+\theta, p}(0, T ; X) \cap C^{1}\left([0, T] ;(X, D(A))_{\theta+1-1 / p, p}\right) .
$$

Since (I-1) is stronger than (I), in view of Theorem A a solution $u$ of (0.1) exists and satisfies (0.2) and (0.3). Set

$$
\varphi_{0}^{\prime}=A \varphi_{0}+A_{1} \varphi_{1}(-r)+\int_{-r}^{0} a(s) A_{2} \varphi_{1}(s) d s+f(0) .
$$


Then (I-1) implies

$$
\varphi_{0}^{\prime} \in(X, D(A))_{\theta+1-1 / p, p}, \quad \varphi_{1}^{\prime} \in W^{\theta, p}(-r, 0 ; D(A)), \quad f^{\prime} \in W^{\theta, p}(0, T ; X) .
$$

Namely, (I) is satisfied by $\varphi_{0}^{\prime}, \varphi_{1}^{\prime}, f^{\prime}$ instead of $\varphi_{0}, \varphi_{1}, f$. Therefore there exists a unique solution $v$ of the following problem:

$$
\left\{\begin{array}{l}
v^{\prime}(t)=A v(t)+A_{1} v(t-r)+\int_{-r}^{0} a(s) A_{2} v(t+s) d s+f^{\prime}(t), \quad 0 \leq t \leq T, \\
v(0)=\varphi_{0}^{\prime}, \quad v(s)=\varphi_{1}^{\prime}(s) \text { a.e. } s \in(-r, 0)
\end{array}\right.
$$

satisfying

$$
\left\{\begin{array}{l}
v \in W^{\theta, p}(0, T ; D(A)) \cap C\left([0, T] ;(X, D(A))_{\theta+1-1 / p, p}\right), \\
d v / d t \in W^{\theta, p}(0, T ; X) .
\end{array}\right.
$$

Set $w(t)=\varphi_{0}+\int_{0}^{t} v(\tau) d \tau$. Then in view of (1.4)

$$
\left\{\begin{array}{l}
w^{\prime} \in W^{\theta, p}(0, T ; D(A)) \cap C\left([0, T] ;(X, D(A))_{\theta+1-1 / p, p}\right), \\
w^{\prime \prime} \in W^{\theta, p}(0, T ; X) .
\end{array}\right.
$$

Since $\varphi_{0} \in D(A), v \in W^{\theta, p}(0, T ; D(A)) \subset L^{p}(0, T ; D(A))$, one has

$$
w(\cdot)=\varphi_{0}+\int_{0} v(\tau) d \tau \in C([0, T] ; D(A)) \subset L^{p}(0, T ; D(A)) .
$$

In view of $(1.5) w^{\prime} \in W^{\theta, p}(0, T ; D(A)) \subset L^{p}(0, T ; D(A))$. By virtue of this and (1.6)

$$
w \in W^{1, p}(0, T ; D(A)) \subset W^{\theta, p}(0, T ; D(A)) .
$$

It follows from (1.7) and (1.5) that

$$
w \in W^{1+\theta, p}(0, T ; D(A)) \cap W^{2+\theta, p}(0, T ; X) .
$$

Since $D(A) \subset(X, D(A))_{\theta+1-1 / p, p}$, one has

$$
w \in C([0, T] ; D(A)) \subset C\left([0, T] ;(X, D(A))_{\theta+1-1 / p, p}\right) .
$$

From this and (1.5) it follows

$$
w \in C^{1}\left([0, T] ;(X, D(A))_{\theta+1-1 / p, p}\right) .
$$

We are going to show

$$
w(t)=u(t), \quad 0 \leq t \leq T .
$$

If this is proved, then (1.1) follows from (1.7) and (1.8).

Set $\breve{v}(t)=\left\{\begin{array}{ll}v(t) & 0 \leq t \leq T, \\ \varphi_{1}^{\prime}(t) & -r \leq t \leq 0 .\end{array}\right.$ Then, $\breve{v} \in W^{\theta, p}(-r, T ; D(A))$. Problem (1.3) is transformed to the following integral equation:

$$
\begin{aligned}
& v(t)=e^{t A} \varphi_{0}^{\prime}+\int_{0}^{t} e^{(t-s) A} A_{1} \breve{v}(s-r) d s+\int_{0}^{t} e^{(t-s) A} \int_{-r}^{0} a(\sigma) A_{2} \breve{v}(s+\sigma) d \sigma d s \\
& +\int_{0}^{t} e^{(t-s) A} f^{\prime}(s) d s .
\end{aligned}
$$


This implies

$$
\begin{aligned}
& \int_{0}^{t} v(\tau) d \tau=\int_{0}^{t} e^{\tau A} \varphi_{0}^{\prime} d \tau+\int_{0}^{t} \int_{0}^{\tau} e^{(\tau-s) A} A_{1} \breve{v}(s-r) d s d \tau \\
& +\int_{0}^{t} \int_{0}^{\tau} e^{(\tau-s) A} \int_{-r}^{0} a(\sigma) A_{2} \breve{v}(s+\sigma) d \sigma d s d \tau+\int_{0}^{t} \int_{0}^{\tau} e^{(\tau-s) A} f^{\prime}(s) d s d \tau
\end{aligned}
$$

(i) Case $T \leq r$. In view of the definition (1.2) of $\varphi_{0}^{\prime}$ one observes

$$
\begin{aligned}
& \int_{0}^{t} e^{\tau A} \varphi_{0}^{\prime} d \tau=\left[e^{t A}-I\right] A^{-1} \varphi_{0}^{\prime} \\
& =e^{t A} \varphi_{0}-\varphi_{0}+\left[e^{t A}-I\right] A^{-1}\left(A_{1} \varphi_{1}(-r)+\int_{-r}^{0} a(s) A_{2} \varphi_{1}(s) d s+f(0)\right) .
\end{aligned}
$$

With the aid of a change of the order of integration and an integration by parts

$$
\begin{aligned}
& \int_{0}^{t} \int_{0}^{\tau} e^{(\tau-s) A} A_{1} \breve{v}(s-r) d s d \tau=\int_{0}^{t} \int_{0}^{\tau} e^{(\tau-s) A} A_{1} \varphi_{1}^{\prime}(s-r) d s d \tau \\
& =\int_{0}^{t}\left[e^{(t-s) A}-I\right] A^{-1} A_{1} \varphi_{1}^{\prime}(s-r) d s \\
& =-\left[e^{t A}-I\right] A^{-1} A_{1} \varphi_{1}(-r)+\int_{0}^{t} e^{(t-s) A} A_{1} \varphi_{1}(s-r) d s .
\end{aligned}
$$

Again changing the order of integration and integrating by parts one obtains

$$
\begin{aligned}
& \int_{0}^{t} \int_{0}^{\tau} e^{(\tau-s) A} \int_{-r}^{0} a(\sigma) A_{2} \breve{v}(s+\sigma) d \sigma d s d \tau=\int_{0}^{t} \int_{s}^{t} e^{(\tau-s) A} \int_{-r}^{0} a(\sigma) A_{2} \breve{v}(s+\sigma) d \sigma d \tau d s \\
& =\int_{0}^{t} \int_{-r}^{0} a(\sigma) \int_{s}^{t} e^{(\tau-s) A} d \tau A_{2} \breve{v}(s+\sigma) d \sigma d s=\int_{0}^{t} \int_{-r}^{0} a(\sigma)\left[e^{(t-s) A}-I\right] A^{-1} A_{2} \breve{v}(s+\sigma) d \sigma d s \\
& =\int_{0}^{t}\left[e^{(t-s) A}-I\right] A^{-1} \int_{-r}^{-s} a(\sigma) A_{2} \varphi_{1}^{\prime}(s+\sigma) d \sigma d s \\
& +\int_{0}^{t}\left[e^{(t-s) A}-I\right] A^{-1} \int_{-s}^{0} a(\sigma) A_{2} v(s+\sigma) d \sigma d s=\mathrm{I}_{1}+\mathrm{I}_{2},
\end{aligned}
$$

where

$$
\begin{aligned}
& \mathrm{I}_{1}=\int_{0}^{t}\left[e^{(t-s) A}-I\right] A^{-1} \int_{-r}^{-s} a(\sigma) A_{2} \varphi_{1}^{\prime}(s+\sigma) d \sigma d s, \\
& \mathrm{I}_{2}=\int_{0}^{t}\left[e^{(t-s) A}-I\right] A^{-1} \int_{-s}^{0} a(\sigma) A_{2} v(s+\sigma) d \sigma d s .
\end{aligned}
$$


Changing the order of integration and integrating by parts yield

$$
\begin{aligned}
\mathrm{I}_{1}=\int_{-r}^{-t} a(\sigma) \int_{0}^{t}\left[e^{(t-s) A}-I\right] A^{-1} A_{2} \varphi_{1}^{\prime}(s+\sigma) d s d \sigma \\
+\int_{-t}^{0} a(\sigma) \int_{0}^{-\sigma}\left[e^{(t-s) A}-I\right] A^{-1} A_{2} \varphi_{1}^{\prime}(s+\sigma) d s d \sigma \\
=\int_{-r}^{-t} a(\sigma)\left\{-\left[e^{t A}-I\right] A^{-1} A_{2} \varphi_{1}(\sigma)+\int_{0}^{t} e^{(t-s) A} A_{2} \varphi_{1}(s+\sigma) d s\right\} d \sigma \\
+\int_{-t}^{0} a(\sigma)\left\{\left[e^{(t+\sigma) A}-I\right] A^{-1} A_{2} \varphi_{1}(0)-\left[e^{t A}-I\right] A^{-1} A_{2} \varphi_{1}(\sigma)\right\} d \sigma \\
+\int_{-t}^{0} a(\sigma)\left\{\int_{0}^{-\sigma} e^{(t-s) A} A_{2} \varphi_{1}(s+\sigma) d s\right\} d \sigma \\
=-\int_{-r}^{0} a(\sigma)\left[e^{t A}-I\right] A^{-1} A_{2} \varphi_{1}(\sigma) d \sigma+\int_{-r}^{-t} a(\sigma) \int_{0}^{t} e^{(t-s) A} A_{2} \varphi_{1}(s+\sigma) d s d \sigma \\
+\int_{-t}^{0} a(\sigma)\left[e^{(t+\sigma) A}-I\right] A^{-1} A_{2} \varphi_{1}(0) d \sigma+\int_{-t}^{0} a(\sigma) \int_{0}^{-\sigma} e^{(t-s) A} A_{2} \varphi_{1}(s+\sigma) d s d \sigma .
\end{aligned}
$$

The sum of the second and fourth terms of the last side of the above equalities is equal to

$$
\begin{aligned}
& \int_{0}^{t} e^{(t-s) A} \int_{-r}^{-t} a(\sigma) A_{2} \varphi_{1}(s+\sigma) d \sigma d s+\int_{0}^{t} e^{(t-s) A} \int_{-t}^{-s} a(\sigma) A_{2} \varphi_{1}(s+\sigma) d \sigma d s \\
& =\int_{0}^{t} e^{(t-s) A} \int_{-r}^{-s} a(\sigma) A_{2} \varphi_{1}(s+\sigma) d \sigma d s .
\end{aligned}
$$

Therefore

$$
\begin{aligned}
& \mathrm{I}_{1}=-\int_{-r}^{0} a(\sigma)\left[e^{t A}-I\right] A^{-1} A_{2} \varphi_{1}(\sigma) d \sigma+\int_{-t}^{0} a(\sigma)\left[e^{(t+\sigma) A}-I\right] A^{-1} A_{2} \varphi_{1}(0) d \sigma \\
& +\int_{0}^{t} e^{(t-s) A} \int_{-r}^{-s} a(\sigma) A_{2} \varphi_{1}(s+\sigma) d \sigma d s .
\end{aligned}
$$

We can show without difficulty

$$
\begin{aligned}
& \mathrm{I}_{2}=\int_{-t}^{0} a(\sigma) \int_{-\sigma}^{t}\left[e^{(t-s) A}-I\right] A^{-1} A_{2} v(s+\sigma) d s d \sigma \\
& =\int_{-t}^{0} a(\sigma) \int_{-\sigma}^{t}\left[e^{(t-s) A}-I\right] A^{-1} \frac{\partial}{\partial s} \int_{0}^{s+\sigma} A_{2} v(\tau) d \tau d s d \sigma \\
& =\int_{-t}^{0} a(\sigma) \int_{-\sigma}^{t} e^{(t-s) A} \int_{0}^{s+\sigma} A_{2} v(\tau) d \tau d s d \sigma \\
& =\int_{-t}^{0} a(\sigma) \int_{-\sigma}^{t} e^{(t-s) A} A_{2}\left(\varphi_{0}+\int_{0}^{s+\sigma} v(\tau) d \tau\right) d s d \sigma-\int_{-t}^{0} a(\sigma) \int_{-\sigma}^{t} e^{(t-s) A} A_{2} \varphi_{0} d s d \sigma \\
& =\int_{-t}^{0} a(\sigma) \int_{-\sigma}^{t} e^{(t-s) A} A_{2}\left(\varphi_{0}+\int_{0}^{s+\sigma} v(\tau) d \tau\right) d s d \sigma \\
& +\int_{-t}^{0} a(\sigma)\left[I-e^{(t+\sigma) A}\right] A^{-1} A_{2} \varphi_{0} d \sigma .
\end{aligned}
$$


From $(1.14)-(1.16)$ and $\varphi_{0}=\varphi_{1}(0)$ it follows that

$$
\begin{aligned}
& \int_{0}^{t} \int_{0}^{\tau} e^{(\tau-s) A} \int_{-r}^{0} a(\sigma) A_{2} v(s+\sigma) d \sigma d s d \tau=\mathrm{I}_{1}+\mathrm{I}_{2} \\
& =-\int_{-r}^{0} a(\sigma)\left[e^{t A}-I\right] A^{-1} A_{2} \varphi_{1}(\sigma) d \sigma+\int_{0}^{t} e^{(t-s) A} \int_{-r}^{-s} a(\sigma) A_{2} \varphi_{1}(s+\sigma) d \sigma d s \\
& +\int_{-t}^{0} a(\sigma) \int_{-\sigma}^{t} e^{(t-s) A} A_{2}\left(\varphi_{0}+\int_{0}^{s+\sigma} v(\tau) d \tau\right) d s d \sigma
\end{aligned}
$$

As is easily seen

$$
\begin{aligned}
& \int_{0}^{t} \int_{0}^{\tau} e^{(\tau-s) A} f^{\prime}(s) d s d \tau=\int_{0}^{t} \int_{s}^{t} e^{(\tau-s) A} d \tau f^{\prime}(s) d s=\int_{0}^{t}\left[e^{(t-s) A}-I\right] A^{-1} f^{\prime}(s) d s \\
& =-\left[e^{t A}-I\right] A^{-1} f(0)+\int_{0}^{t} e^{(t-s) A} f(s) d s .
\end{aligned}
$$

From $(1.11)-(1.13),(1.17),(1.18)$ the following equality follows easily:

$$
\begin{aligned}
& w(t)=e^{t A} \varphi_{0}+\int_{0}^{t} e^{(t-s) A} A_{1} \varphi_{1}(s-r) d s+\int_{0}^{t} e^{(t-s) A} \int_{-r}^{-s} a(\sigma) A_{2} d \sigma d s \\
& +\int_{-t}^{0} a(\sigma) \int_{-\sigma}^{t} e^{(t-s) A} A_{2} w(s+\sigma) d s d \sigma+\int_{0}^{t} e^{(t-s) A} f(s) d s
\end{aligned}
$$

Set $\widehat{w}(t)=\left\{\begin{array}{ll}w(t) & 0 \leq t \leq T, \\ \varphi_{1}(t) & -r \leq t \leq 0 .\end{array}\right.$ Then (1.19) is rewritten as

$$
\begin{aligned}
& w(t)=e^{t A} \varphi_{0}+\int_{0}^{t} e^{(t-s) A} A_{1} \widehat{w}(s-r) d s+\int_{0}^{t} e^{(t-s) A} \int_{-r}^{0} a(\sigma) A_{2} \widehat{w}(s+\sigma) d \sigma d s \\
& +\int_{0}^{t} e^{(t-s) A} f(s) d s .
\end{aligned}
$$

Consequently (1.9) is obtained.

(ii) Case $r<T \leq 2 r$. By virtue of the result established in the previous case $0<T \leq r$ we already know that $w(t)=u(t)$ for $0 \leq t \leq r$. Hence

$$
w(t)=\varphi_{0}+\int_{0}^{r} v(\tau) d \tau+\int_{r}^{t} v(\tau) d \tau=w(r)+\int_{r}^{t} v(\tau) d \tau=u(r)+\int_{r}^{t} v(\tau) d \tau .
$$

Since

$$
A u(r)+A_{1} u(0)+\int_{-r}^{0} a(s) A_{2} u(r+s) d s=u^{\prime}(r)-f(r) \in(X, D(A))_{\theta+1-1 / p, p},
$$

the following facts hold:

$$
\left\{\begin{array}{l}
u_{[0, r]} \in W^{1+\theta, p}(0, r ; D(A)), \\
f \in W^{1+\theta, p}(r, T ; X) \cap C\left([r, T] ;(X, D(A))_{\theta+1-1 / p, p}\right), \\
A u_{[0, r]}(r)+A_{1} u_{[0, r]}(0)+\int_{-r}^{0} a(s) A_{2} u_{[0, r]}(r+s) d s \in(X, D(A))_{\theta+1-1 / p, p} .
\end{array}\right.
$$


Hence (I- 1$)$ is satisfied with $[-r, 0], \varphi_{1}$ replaced by $[0, r], u_{[0, r]}$ respectively. Therefore, by the method of the previous case we can show $w(t)=u(t)$ for $r \leq t \leq T$.

We can proceed to show (1.9) in the general case, and the proof of Theorem 1 is complete.

In case $1 / p<\theta<1$ we assume

$$
\left\{\begin{array}{l}
\varphi_{1} \in W^{1+\theta, p}(-r, 0 ; D(A)) \quad\left(\Longrightarrow \varphi_{1}(0) \in D(A)\right), \\
\left.f \in W^{1+\theta, p}(0, T ; X) \cap C^{1}[0, T] ;(X, D(A))_{\theta-1 / p, p}\right), \\
A \varphi_{1}(0)+A_{1} \varphi_{1}(-r)+\int_{-r}^{0} a(s) A_{2} \varphi_{1}(s) d s \in(X, D(A))_{\theta-1 / p, p}, \\
A \varphi_{1}^{\prime}(0)+A_{1} \varphi_{1}^{\prime}(-r)+\int_{-r}^{0} a(s) A_{2} \varphi_{1}^{\prime}(s) d s \in(X, D(A))_{\theta-1 / p, p}, \\
\varphi_{1}^{\prime}(0)\left(=D_{-} \varphi_{1}(0)\right)=A \varphi_{1}(0)+A_{1} \varphi_{1}(-r)+\int_{-r}^{0} a(\sigma) A_{2} \varphi_{1}(\sigma) d \sigma+f(0) .
\end{array}\right.
$$

Theorem 2. Suppose $1 / p<\theta<1$. If assumption (II-1) is satisfied, the solution $u$ of (0.1) satisfies

$$
u \in W^{1+\theta, p}(0, T ; D(A)) \cap W^{2+\theta, p}(0, T ; X) \cap C^{2}\left([0, T] ;(X, D(A))_{\theta-1 / p, p}\right) .
$$

If hypothesis (II-1) holds, then (II) is satisfied by $\varphi_{1}^{\prime}$ and $f^{\prime}$ in place of $\varphi_{1}$ and $f$ respectively. Therefore according to Theorem B there exists a unique solution $v$ of the following problem

$$
\begin{aligned}
& \frac{d}{d t} v(t)=A v(t)+A_{1} v(t-r)+\int_{-r}^{0} a(s) A_{2} v(t+s) d s+f^{\prime}(t) \\
& v(s)=\varphi_{1}^{\prime}(s), \quad-r \leq s \leq 0
\end{aligned}
$$

satisfying

$$
\begin{aligned}
& v \in W^{\theta, p}(0, T ; D(A)), \\
& d v / d t \in W^{\theta, p}(0, T ; X) \cap C\left([0, T] ;(X, D(A))_{\theta-1 / p}\right) .
\end{aligned}
$$

Set

$$
w(t)=\varphi_{0}+\int_{0}^{t} v(\tau) d \tau .
$$

In view of $(1.23),(1.24)$ one has

$$
\begin{aligned}
& w^{\prime} \in W^{\theta, p}(0, T ; D(A)), \\
& w^{\prime \prime} \in W^{\theta, p}(0, T ; X) \cap C\left([0, T] ;(X, D(A))_{\theta-1 / p, p}\right) .
\end{aligned}
$$

Since $\theta>1 / p, W^{\theta, p}(0, T ; D(A)) \subset C([0, T] ; D(A))$. Hence $v \in C([0, T] ; D(A))$. From this and $\varphi_{0} \in D(A)$ it follows that $w \in C^{1}([0, T] ; D(A))$. This implies $w \in W^{1, p}(0, T ; D(A)) \subset$ $W^{\theta, p}(0, T ; D(A))$. Hence with the aid of $(1.26),(1.27)$ we deduce

$$
w \in W^{1+\theta, p}(0, T ; D(A)) \cap W^{2+\theta, p}(0, T ; X) .
$$


Since $D(A) \subset(X, D(A))_{\theta-1 / p, p}$, one also has $w \in C^{1}\left([0, T] ;(X, D(A))_{\theta-1 / p, p}\right)$. From this and (1.27) it follows that

$$
w \in C^{2}\left([0, T] ;(X, D(A))_{\theta-1 / p, p}\right)
$$

If it is shown that $w(t)=u(t), 0 \leq t \leq T$, then in view of (1.28) and (1.29) the proof of Theorem 2 is complete. This part of the proof is almost the same as that of Theorem 1 , and so it is omitted.

\section{Solution Semigroup: Case $\theta<1 / p$}

Suppose assumption (I) is satisfied. Set

$$
Z=(X, D(A))_{\theta+1-1 / p, p} \times W^{\theta, p}(-r, 0 ; D(A)) .
$$

Following G. Di Blasio, K. Kunisch and E. Sinestrari [3] the solution semigroup for (0.1) is defined as follows:

$$
S(t)\left(\begin{array}{c}
\varphi_{0} \\
\varphi_{1}
\end{array}\right)=\left(\begin{array}{c}
u(t) \\
\widehat{u}_{t}
\end{array}\right), t \geq 0, \text { for }\left(\begin{array}{c}
\varphi_{0} \\
\varphi_{1}
\end{array}\right) \in Z,
$$

where $u$ is the solution of problem $(0.1)$ with $f(t) \equiv 0$, and $\widehat{u}(t)= \begin{cases}u(t) & 0 \leq t<\infty, \\ \varphi_{1}(t) & -r \leq t \leq 0,\end{cases}$ $\widehat{u}_{t}(s)=\widehat{u}(t+s)$ for $-r \leq s \leq 0$. Since $u \in W^{\theta, p}(0, \infty ; D(A)) \cap$ $C\left([0, \infty) ;(X, D(A))_{\theta+1-1 / p, p}\right)$, where $u \in W^{\theta, p}(0, \infty ; D(A))$ means $u \in W^{\theta, p}(0, T ; D(A))$ for any $0<T<\infty, u(t) \in(X, D(A))_{\theta+1-1 / p, p}$ for $t \geq 0, \widehat{u} \in W^{\theta, p}(-r, \infty ; D(A))$, and hence $\widehat{u}_{t} \in W^{\theta, p}(-r, 0 ; D(A))$ for $0 \leq t<\infty$. Therefore $S(t): Z \mapsto Z$ and $S(0)=I$. It can be shown without difficulty that $S(t)$ is a $C_{0}$-semigroup in $Z$.

Theorem 3. The infinitesimal generater of the solution semigroup $S(t)$ is given by

$$
\begin{aligned}
& D(\Lambda)=\left\{\left(\begin{array}{c}
\varphi_{0} \\
\varphi_{1}
\end{array}\right) ; \varphi_{1} \in W^{1+\theta, p}(-r, 0 ; D(A)), \varphi_{1}(0)=\varphi_{0},\right. \\
& \left.A \varphi_{0}+A_{1} \varphi_{1}(-r)+\int_{-r}^{0} a(s) A_{2} \varphi_{1}(s) d s \in(X, D(A))_{\theta+1-1 / p, p}\right\}, \\
& \Lambda\left(\begin{array}{c}
\varphi_{0} \\
\varphi_{1}
\end{array}\right)=\left(\begin{array}{c}
A \varphi_{0}+A_{1} \varphi_{1}(-r)+\int_{-r}^{0} a(s) A_{2} \varphi_{1}(s) d s \\
\varphi_{1}^{\prime}
\end{array}\right) .
\end{aligned}
$$

This theorem can be establised by showing the following statements following G. Di Blasio, K. Kunisch and E. Sinestrari [3]:

(i) $S(t) D(\Lambda) \subset D(\Lambda)$,

(ii) $D(\Lambda)$ is dense in $Z$,

(iii) $\Lambda \subset$ infinitesimal generator of $\{S(t)\}$,

(iv) $\Lambda: D(\Lambda) \subset Z \rightarrow Z$ is closed.

Problem (0.1) is rewritten as

$$
\left\{\begin{array}{l}
\frac{d}{d t}\left(\begin{array}{c}
u(t) \\
\widehat{u}_{t}
\end{array}\right)=\Lambda\left(\begin{array}{c}
u(t) \\
\widehat{u}_{t}
\end{array}\right)+\left(\begin{array}{c}
f(t) \\
0
\end{array}\right), 0 \leq t \leq T \\
\left(\begin{array}{c}
u(0) \\
\widehat{u}_{0}
\end{array}\right)=\left(\begin{array}{c}
\varphi_{0} \\
\varphi_{1}
\end{array}\right) .
\end{array}\right.
$$


The mild solution of (2.2) is expressed as

$$
\left(\begin{array}{c}
u(t) \\
\widehat{u}_{t}
\end{array}\right)=S(t)\left(\begin{array}{c}
\varphi_{0} \\
\varphi_{1}
\end{array}\right)+\int_{0}^{t} S(t-s)\left(\begin{array}{c}
f(s) \\
0
\end{array}\right) d s
$$

If $\left(\begin{array}{c}\varphi_{0} \\ \varphi_{1}\end{array}\right) \in D(\Lambda)$ and $f \in C^{1}\left([0, T] ;(X, D(A))_{\theta+1-1 / p, p}\right)$, then $\left(\begin{array}{c}u(t) \\ \widehat{u}_{t}\end{array}\right)$ is a strict solution, and

$$
\begin{aligned}
& u \in C^{1}\left([0, T] ;(X, D(A))_{\theta+1-1 / p, p}\right), \\
& \widehat{u} . \in C^{1}\left([0, T] ; W^{\theta, p}(-r, 0 ; D(A))\right), \\
& \frac{d}{d t}\left(\begin{array}{c}
u(t) \\
\widehat{u}_{t}
\end{array}\right)=S(t) \Lambda\left(\begin{array}{c}
\varphi_{0} \\
\varphi_{1}
\end{array}\right)+S(t)\left(\begin{array}{c}
f(0) \\
0
\end{array}\right)+\int_{0}^{t} S(t-s)\left(\begin{array}{c}
f^{\prime}(s) \\
0
\end{array}\right) d s .
\end{aligned}
$$

Starting from

$$
u . \in C\left([0, T] ; L^{p}(-r, 0 ; D(A))\right) \Longleftrightarrow u \in L^{p}(-r, T ; D(A))
$$

one can show that $(2.3)$ is equivalent to $\widehat{u} \in W^{1+\theta, p}(-r, T ; D(A))$. Thus the following assertion holds:

Theorem 4. If the following assumptions are satisfied:

$$
\begin{aligned}
& \varphi_{1} \in W^{1+\theta, p}(-r, 0 ; D(A)), \quad \varphi_{0}=\varphi_{1}(0), \quad f \in C^{1}\left([0, T] ;(X, D(A))_{\theta+1-1 / p, p}\right), \\
& A \varphi_{0}+A_{1} \varphi_{1}(-r)+\int_{-r}^{0} a(s) A_{2} \varphi_{1}(s) d s \in(X, D(A))_{\theta+1-1 / p, p},
\end{aligned}
$$

then a solution of (0.1) satisfying

$$
u \in W^{1+\theta, p}(0, T ; D(A)) \cap C^{1}\left([0, T] ;(X, D(A))_{\theta+1-1 / p, p}\right)
$$

exists and is unique.

\section{Regularity of Solutions: Case $\theta>1 / p$}

In this section we suppose that the following assumptions are satisfied:

$$
\begin{aligned}
& A_{1} A^{-1}, A_{2} A^{-1} \in \mathcal{L}\left((X, D(A))_{\theta-1 / p, p},(X, D(A))_{\theta-1 / p, p}\right) \\
& \left\{\begin{array}{l}
\varphi_{1} \in W^{\theta, p}(-r, 0 ; D(A)) \cap C\left([-r, 0] ;(X, D(A))_{\theta+1-1 / p, p}\right) \\
f \in W^{\theta, p}(0, T ; X) \cap C\left([0, T] ;(X, D(A))_{\theta-1 / p, p}\right)
\end{array}\right.
\end{aligned}
$$

Remark 1. Set $\varphi_{0}=\varphi_{1}(0)$. Then it follows from (II-3) that $\varphi_{0} \in(X, D(A))_{\theta+1-1 / p, p}$. Hence $A \varphi_{0} \in(X, D(A))_{\theta-1 / p, p}$. From $\varphi_{1} \in C\left([-r, 0] ;(X, D(A))_{\theta+1-1 / p, p}\right)$ it follows that

$$
A \varphi_{1} \in C\left([-r, 0] ;(X, D(A))_{\theta-1 / p, p}\right), \quad \int_{-r}^{0} a(s) A \varphi_{1}(s) d s \in(X, D(A))_{\theta-1 / p, p} .
$$

Hence by (II-2)

$$
A_{1} \varphi_{1} \in C\left([-r, 0] ;(X, D(A))_{\theta-1 / p, p}\right), \quad \int_{-r}^{0} a(s) A_{2} \varphi_{1}(s) d s \in(X, D(A))_{\theta-1 / p, p} .
$$


Hence the final condition of (II) is satisfied. Therefore (II-2) and (II-3) imply (II).

Remark 2. (II-2) is equivalent to

$$
A_{i} \in \mathcal{L}\left((X, D(A))_{\theta+1-1 / p, p},(X, D(A))_{\theta-1 / p, p}\right), i=1,2 .
$$

A comment on assumption (II-2) will be given in the final section.

Theorem 5. Suppose $\theta>1 / p$, and assumptions (II-2) and (II-3) are satisfied. Then the solution $u$ of problem (0.1) satisfies

$$
\begin{aligned}
& u \in W^{\theta, p}(0, T ; D(A)) \cap C\left([0, T] ;(X, D(A))_{\theta+1-1 / p, p}\right), \\
& d u / d t \in W^{\theta, p}(0, T ; X) \cap C\left([0, T] ;(X, D(A))_{\theta-1 / p, p}\right) .
\end{aligned}
$$

Suppose first $T \leq r$. Let $u_{0}$ be the function defined by

$$
u_{0}(t)=e^{t A}\left[\varphi_{1}(0)+A^{-1} \tilde{f}(0)\right]+\int_{0}^{t} e^{(t-s) A} \widetilde{f}_{*}(s) d s-A^{-1} \widetilde{f}(0),
$$

where

$$
\begin{aligned}
& \tilde{f}(s)=A_{1} \varphi_{1}(s-r)+f(s)+\int_{-r}^{0} a(\sigma) A_{2} \varphi_{1}(\sigma) d \sigma, \\
& \tilde{f}_{*}(s)=\widetilde{f}(s)-\widetilde{f}(0)=A_{1} \varphi_{1}(s-r)+f(s)-A_{1} \varphi_{1}(-r)-f(0) .
\end{aligned}
$$

It follows from (II-3) and (3.1) that

$$
\begin{aligned}
& \tilde{f} \in W^{\theta, p}(0, T ; X) \cap C\left([0, T] ;(X, D(A))_{\theta-1 / p, p}\right), \\
& \widetilde{f}_{*} \in W_{*}^{\theta, p}(0, T ; X) \cap C\left([0, T] ;(X, D(A))_{\theta-1 / p, p}\right), \\
& \varphi_{0}+A^{-1} \widetilde{f}(0) \in(X, D(A))_{\theta+1-1 / p, p} .
\end{aligned}
$$

The solution of $(0.1)$ is obtained as the solution of the following integral equation

$$
u(t)=u_{0}(t)+\int_{0}^{t} e^{(t-s) A} \int_{-r}^{0} a(\sigma)\left[A_{2} \widehat{u}(s+\sigma)-A_{2} \varphi_{1}(\sigma)\right] d \sigma d s,
$$

where $\widehat{u}(s)=\left\{\begin{array}{ll}u(s) & s \geq 0, \\ \varphi_{1}(s) & s<0 .\end{array}\right.$ This equation is sovled by successive approximation:

$$
u_{n+1}(t)=u_{0}(t)+\int_{0}^{t} e^{(t-s) A} \int_{-r}^{0} a(\sigma)\left[A_{2} \widehat{u}_{n}(s+\sigma)-A_{2} \varphi_{1}(\sigma)\right] d \sigma d s, n=1,2,3, \ldots
$$

It is shown in A. Favini and H. Tanabe [2] that $u_{n} \in W^{\theta, p}(0, T ; D(A)), u_{n}(0)=\varphi_{0}$, $n=0,1,2, \ldots$.. From (3.9) it follows that

$$
u_{n+1}(t)-u_{n}(t)=\int_{0}^{t} e^{(t-s) A} \int_{-r}^{0} a(\sigma)\left[A_{2} \widehat{u}_{n}(s+\sigma)-A_{2} \widehat{u}_{n-1}(s+\sigma)\right] d \sigma d s .
$$

If $-r \leq \sigma<-T$, then $s+\sigma<t-T \leq 0$. Hence

$$
A_{2} \widehat{u}_{n}(s+\sigma)-A_{2} \widehat{u}_{n-1}(s+\sigma)=\varphi_{1}(s+\sigma)-\varphi_{1}(s+\sigma)=0 .
$$


Therefore (3.10) is rewritten as

$$
u_{n+1}(t)-u_{n}(t)=\int_{0}^{t} e^{(t-s) A} \int_{-T}^{0} a(\sigma)\left[A_{2} \widehat{u}_{n}(s+\sigma)-A_{2} \widehat{u}_{n-1}(s+\sigma)\right] d \sigma d s .
$$

It is proved in [2] that

$$
\left\|u_{n+1}-u_{n}\right\|_{W^{\theta, p}(0, T ; D(A))} \leq C_{2}^{\prime} k_{2}\|a\|_{L^{1}(-T, 0)}\left[(\theta p)^{-1 / p} C_{0}^{\prime}+1\right]\left\|u_{n}-u_{n-1}\right\|_{W^{\theta, p}(0, T ; D(A))}
$$

for some constants $C_{0}^{\prime}, C_{2}^{\prime}$ independent of $T$ and $k_{2}=\left\|A_{2} A^{-1}\right\|$. Therefore if $T$ is so small that

$$
C_{2}^{\prime} k_{2}\|a\|_{L^{1}(-T, 0)}\left[(\theta p)^{-1 / p} C_{0}^{\prime}+1\right]<1,
$$

then

$$
\sum_{n=1}^{\infty}\left\|u_{n+1}-u_{n}\right\|_{W^{\theta, p}(0, T ; D(A))}<\infty .
$$

Set

$$
W_{*}^{\theta, p}(0, T ; X)=\left\{u \in W^{\theta, p}(0, T ; X) ; u(0)=0\right\} .
$$

The following lemma is due to G. Di Blasio [5] (Theorem 10 if $\theta<1 / p$ and Theorem 8 if $\theta>1 / p)$. Also c.f. Lemma 1 of G. Di Blasio and A. Lorenzi [1].

Lemma 1. Suppose $\theta \neq 1 / p$. If $x \in(X, D(A))_{\theta+1-1 / p, p}$, then

$$
e^{-\cdot A} x \in W^{\theta, p}(0, T ; D(A)) \cap C\left([0, T] ;(X, D(A))_{\theta+1-1 / p, p}\right) .
$$

The following lemma is Theorem 24 of G. Di Blasio [5].

Lemma 2. Suppose $\theta>1 / p$. Then, if $f \in W_{*}^{\theta, p}(0, T ; X)$, the function $V(t)=$ $\int_{0}^{t} e^{(t-s) A} f(s) d s$ satisfies

$$
\begin{aligned}
& V \in W^{\theta, p}(0, T ; D(A)), \\
& d V / d t=A V+f \in C\left([0, T] ;(X, D(A))_{\theta-1 / p, p}\right),
\end{aligned}
$$

and the following inequality holds with a constant $C_{2}^{\prime}$ independent of $T$ :

$$
\|V\|_{W^{\theta, p}(0, T ; D(A))} \leq C_{2}^{\prime}\|f\|_{W^{\theta, p}(0, T ; X)} .
$$

In A. Favini and H. Tanabe [2] it is shown that the constant $C_{2}^{\prime}$ above can be chosen independent of $T$ if we choose (0.6) as the norm of $W^{\theta, p}(0, T ; D(A))$.

Let $V$ and $f$ be as in Lemma 2. With the aid of (3.14) and (3.15) one observes

$$
\begin{aligned}
& \left\|V^{\prime}\right\|_{W^{\theta, p}(0, T ; X)}=\|A V+f\|_{W^{\theta, p}(0, T ; X)} \leq\|V\|_{W^{\theta, p}(0, T ; D(A))}+\|f\|_{W^{\theta, p}(0, T ; X)} \\
& \leq\left(C_{2}^{\prime}+1\right)\|f\|_{W^{\theta, p}(0, T ; X)} .
\end{aligned}
$$

From Lemma 2 the following lemma follows:

Lemma 3. Suppose $\theta>1 / p$. Let $f \in W_{*}^{\theta, p}(0, T ; X) \cap C\left([0, T] ;(X, D(A))_{\theta-1 / p, p}\right)$. Then, for $V(t)=\int_{0}^{t} e^{(t-s) A} f(s) d s$ one has

$$
V \in W^{\theta, p}(0, T ; D(A)) \cap C\left([0, T] ;(X, D(A))_{\theta+1-1 / p, p}\right) .
$$


In view of Lemma 1, 3 and (3.6), (3.7) one observes

$$
\begin{aligned}
& e^{-\cdot A}\left[\varphi_{1}(0)+A^{-1} \widetilde{f}(0)\right] \in C\left([0, T] ;(X, D(A))_{\theta+1-1 / p, p}\right), \\
& \int_{0}^{\cdot} e^{-(\cdot-s) A} \widetilde{f}_{*}(s) d s \in C\left([0, T] ;(X, D(A))_{\theta+1-1 / p, p}\right) .
\end{aligned}
$$

Moreover, $A^{-1} \tilde{f}(0)$ is a constant function with a value in $(X, D(A))_{\theta+1-1 / p, p}$. Consequently

$$
u_{0} \in C\left([0, T] ;(X, D(A))_{\theta+1-1 / p, p}\right) .
$$

Suppose for some $n=1,2, \ldots$

$$
u_{n} \in C\left([0, T] ;(X, D(A))_{\theta+1-1 / p, p}\right) .
$$

Then $\widehat{u}_{n} \in C\left([-r, T] ;(X, D(A))_{\theta+1-1 / p, p}\right)$. Hence $A_{2} \widehat{u}_{n} \in C\left([-r, T] ;(X, D(A))_{\theta-1 / p, p}\right)$ in view of Remark 2 . Therefore it is easy to show

$$
\int_{-r}^{0} a(\sigma)\left[A_{2} \widehat{u}_{n}(\cdot+\sigma)-A_{2} \varphi_{1}(\sigma)\right] d \sigma \in C\left([0, T] ;(X, D(A))_{\theta-1 / p, p}\right) .
$$

Since $u_{n} \in W^{\theta, p}(0, T ; D(A))$, one has $\widehat{u}_{n} \in W^{\theta, p}(-r, T ; D(A))$, and hence $A_{2} \widehat{u}_{n} \in$ $W^{\theta, p}(-r, T ; X)$. The following lemma is proved in A. Favini and H. Tanabe [2, Lemma $2.5]$ :

Lemma 4. Suppose $v \in W^{\theta, p}(-r, T ; X), 0<\theta<1, \theta \neq 1 / p$. Then $\int_{-r}^{0} a(\sigma) v(\cdot+\sigma) d \sigma \in$ $W^{\theta, p}(0, T ; X)$, and

$$
\left\|\int_{-r}^{0} a(\sigma) v(\cdot+\sigma) d \sigma\right\|_{W^{\theta, p}(0, T ; X)} \leq\|a\|_{L^{1}(-r, 0)}\left[N_{\theta, p,(-r, T)}(v)+T^{-\theta}\|v\|_{L^{p}(-r, T ; X)}\right] .
$$

Applying Lemma 4 to $A_{2} \widehat{u}_{n}$ one observes $\int_{-r}^{0} a(\sigma) A_{2} \widehat{u}_{n}(\cdot+\sigma) d \sigma \in W^{\theta, p}(0, T ; X)$. Therefore, noting (3.1) one deduces

$$
\begin{aligned}
& \int_{-r}^{0} a(\sigma)\left[A_{2} \widehat{u}_{n}(\cdot+\sigma)-A_{2} \varphi_{1}(\sigma)\right] d \sigma \\
& =\int_{-r}^{0} a(\sigma) A_{2} \widehat{u}_{n}(\cdot+\sigma) d \sigma-\int_{-r}^{0} a(\sigma) A_{2} \varphi_{1}(\sigma) d \sigma \in W_{*}^{\theta, p}(0, T ; X) .
\end{aligned}
$$

By virtue of (3.19), (3.20) and Lemma 3 one obtains

$$
\int_{0}^{\cdot} e^{(\cdot-s) A} \int_{-r}^{0} a(\sigma)\left[A_{2} \widehat{u}_{n}(s+\sigma)-A_{2} \varphi_{1}(\sigma)\right] d \sigma d s \in C\left([0, T] ;(X, D(A))_{\theta+1-1 / p, p}\right) .
$$

From (3.9), (3.17) and (3.21) it follows that (3.18) holds with $n+1$ in place of $n$.

Next, we estimate the following norm:

$$
\left\|V^{\prime}\right\|_{L^{p}\left(0, T ;(X, D(A))_{\theta, p}\right)}=\left(\left\|V^{\prime}\right\|_{L^{p}(0, T ; X)}^{p}+\int_{0}^{T}\left|V^{\prime}(t)\right|_{\theta, p}^{p} d t\right)^{1 / p}
$$


where $|\cdot|_{\theta, p}$ is the semi norm defined by

$$
|u|_{\theta, p}=\left(\int_{0}^{\infty}\left\|t^{1-\theta} A e^{t A} u\right\|^{p} t^{-1} d t\right)^{1 / p} .
$$

The following inequality was shown in the proof of Lemma 2 of G. Di Blasio [5]:

$$
\left\|V^{\prime}\right\|_{L^{p}(0, T ; X)} \leq((p-1) / \theta p)^{(p-1) / p} M_{1} T^{\theta} N_{\theta, p,(0, T)}(f)+M_{0}\|f\|_{L^{p}(0, T ; X)},
$$

where $M_{0}, M_{1}$ are constants such that $\left\|e^{t A}\right\| \leq M_{0},\left\|(d / d t) e^{t A}\right\| \leq M_{1} / t$. In the proof of Theorem 26 of $[5$, p. 81] it was shown that

$$
\begin{aligned}
& \int_{0}^{T}\left|V^{\prime}(t)\right|_{\theta, p}^{p} d t \leq 2^{4 p-2} M_{1}^{2 p}\left(\theta^{-p}+(1-\theta)^{-p}\right)\left(N_{\theta, p,(0, T)}^{p}(f)+(\theta p)^{-1} \int_{0}^{T} t^{-\theta p}\|f(t)\|^{p} d t\right) \\
& +2^{p-1} M_{1}^{p} \int_{0}^{T} t^{-\theta p}\|f(t)\|^{p} d t \int_{0}^{\infty}(s+1)^{-p} s^{-1+p-p \theta} d s .
\end{aligned}
$$

It is easy to show the following inequality holds for $f \in W_{*}^{\theta, p}(0, T ; X)$ with a constant $c$ independent of $T$ :

$$
\int_{0}^{T} t^{-\theta p}\|f(t)\|^{p} d t \leq c\|f\|_{W^{\theta, p}(0, T ; X)}^{p} .
$$

From (3.22) - (3.24) and (3.25) it follows that the following inequality holds with a constant $C_{1}$ independent of $T$ :

$$
\int_{0}^{T}\left|V^{\prime}(t)\right|_{\theta, p}^{p} d t \leq C_{1}\|f\|_{W^{\theta, p}(0, T ; X)}^{p} .
$$

By virtue of (3.22), (3.23) and (3.26) the following inequality holds for $f \in W_{*}^{\theta, p}(0, T ; X)$ with a constant $C_{2}$ independent of $T$ :

$$
\left\|V^{\prime}\right\|_{L^{p}\left(0, T ;(X, D(A))_{\theta, p}\right)} \leq C_{2}\left(T^{\theta}+1\right)\|f\|_{W^{\theta, p}(0, T ; X)} .
$$

The following lemma is also due to Lemma 11 of G. di Blasio [5].

Lemma 5. Suppose $\theta>p$. Then

$$
W^{\theta, p}(0, T ; X) \cap L^{p}\left(0, T ;(X, D(A))_{\theta, p}\right) \subset C\left([0, T] ;(X, D(A))_{\theta-1 / p, p}\right),
$$

and the following inequality holds for $u \in W^{\theta, p}(0, T ; X) \cap L^{p}\left(0, T ;(X, D(A))_{\theta, p}\right)$ with a constant $C_{3}$ independent of $T$ :

$$
\|u\|_{C\left([0, T] ;(X, D(A))_{\theta-1 / p, p}\right)} \leq C_{3}\left(T^{\theta-1 / p}\|u\|_{W^{\theta, p}(0, T ; X)}+T^{-1 / p}\|u\|_{L^{p}\left(0, T ;(X, D(A))_{\theta, p}\right)}\right) .
$$

Inequality (3.28) follows from the one in case $T=1$ and considering a function $u(T t), 0 \leq t \leq 1$, in the general case.

In view of Lemma 5

$$
\begin{aligned}
& \left\|V^{\prime}\right\|_{C\left([0, T] ;(X, D(A))_{\theta-1 / p, p}\right)} \leq \\
& \leq C_{3}\left(T^{\theta-1 / p}\left\|V^{\prime}\right\|_{W^{\theta, p}(0, T ; X)}+T^{-1 / p}\left\|V^{\prime}\right\|_{L^{p}\left(0, T ;(X, D(A))_{\theta, p}\right)}\right) .
\end{aligned}
$$


Inequalities (3.16), (3.27) and (3.29) yield

$$
\left\|V^{\prime}\right\|_{C\left([0, T] ;(X, D(A))_{\theta-1 / p, p}\right)} \leq C_{T}\|f\|_{W^{\theta, p}(0, T ; X)},
$$

where $C_{T}=C_{3}\left(\left(C_{2}+C_{2}^{\prime}+1\right) T^{\theta}+C_{2}\right) T^{-1 / p}$. Hence

$$
\begin{aligned}
& \|V\|_{C\left([0, T] ;(X, D(A))_{\theta+1-1 / p, p}\right)}=\|A V\|_{C\left([0, T] ;(X, D(A))_{\theta-1 / p, p}\right)} \\
& =\left\|V^{\prime}-f\right\|_{C\left([0, T] ;(X, D(A))_{\theta-1 / p, p}\right)} \leq C_{T}\|f\|_{W^{\theta, p}(0, T ; X)}+\|f\|_{C\left([0, T] ;(X, D(A))_{\theta-1 / p, p}\right)} .
\end{aligned}
$$

We apply $(3.31)$ to $\int_{-T}^{0} a(\sigma)\left[A_{2} \widehat{u}_{n}(\cdot+\sigma)-A_{2} \widehat{u}_{n-1}(\cdot+\sigma)\right] d \sigma$. Then $V=u_{n+1}-u_{n}$ (c.f. (3.11)). Let $T$ satisfy (3.12):

$$
C_{2}^{\prime} k_{2}\|a\|_{L^{1}(-T, 0)}\left[(\theta p)^{-1 / p} C_{0}^{\prime}+1\right]<1 .
$$

One has

$$
\begin{aligned}
& \left\|u_{n+1}-u_{n}\right\|_{C\left([0, T] ;(X, D(A))_{\theta+1-1 / p, p}\right)} \\
& \leq C_{T}\left\|\int_{-T}^{0} a(\sigma)\left[A_{2} \widehat{u}_{n}(\cdot+\sigma)-A_{2} \widehat{u}_{n-1}(\cdot+\sigma)\right] d \sigma\right\|_{W^{\theta, p}(0, T ; X)} \\
& +\left\|\int_{-T}^{0} a(\sigma)\left[A_{2} \widehat{u}_{n}(\cdot+\sigma)-A_{2} \widehat{u}_{n-1}(\cdot+\sigma)\right] d \sigma\right\|_{C\left([0, T] ;(X, D(A))_{\theta-1 / p, p}\right)} .
\end{aligned}
$$

It is shown in [2] that the following inequality holds:

$$
\begin{aligned}
& \left\|\int_{-T}^{0} a(\sigma)\left[A_{2} \widehat{u}_{n}(\cdot+\sigma)-A_{2} \widehat{u}_{n-1}(\cdot+\sigma)\right] d \sigma\right\|_{W^{\theta, p}(0, T ; X)} \\
& \leq k_{2}\|a\|_{L^{1}(-T, 0)}\left[(\theta p)^{-1 / p} C_{0}^{\prime}+1\right]\left\|u_{n}-u_{n-1}\right\|_{W^{\theta, p}(0, T ; D(A))}
\end{aligned}
$$

As is easily seen

$$
\begin{aligned}
& \left\|\int_{-T}^{0} a(\sigma)\left[A_{2} \widehat{u}_{n}(\cdot+\sigma)-A_{2} \widehat{u}_{n-1}(\cdot+\sigma)\right] d \sigma\right\|_{C\left([0, T] ;(X, D(A))_{\theta-1 / p, p}\right)} \\
& =\sup _{s \in[0, T]}\left\|\int_{-T}^{0} a(\sigma)\left[A_{2} \widehat{u}_{n}(s+\sigma)-A_{2} \widehat{u}_{n-1}(s+\sigma)\right] d \sigma\right\|_{(X, D(A))_{\theta-1 / p, p}} \\
& \leq \int_{-T}^{0}|a(\sigma)| d \sigma \sup _{\tau \in[-T, T]}\left\|A_{2} \widehat{u}_{n}(\tau)-A_{2} \widehat{u}_{n-1}(\tau)\right\|_{(X, D(A))_{\theta-1 / p, p}} \\
& \leq k_{2}\|a\|_{L^{1}(-T, 0)} \sup _{\tau \in[0, T]}\left\|A u_{n}(\tau)-A u_{n-1}(\tau)\right\|_{(X, D(A))_{\theta-1 / p, p}} \\
& =k_{2}\|a\|_{L^{1}(-T, 0)}\left\|u_{n}-u_{n-1}\right\|_{C\left([0, T] ;(X, D(A))_{\theta+1-1 / p, p}\right)} .
\end{aligned}
$$

The following inequality follows from (3.32) - (3.34):

$$
\begin{aligned}
& \left\|u_{n+1}-u_{n}\right\|_{C\left([0, T] ;(X, D(A))_{\theta+1-1 / p, p}\right)} \\
& \leq C_{T} k_{2}\|a\|_{L^{1}(-T, 0)}\left[(\theta p)^{-1 / p} C_{0}^{\prime}+1\right]\left\|u_{n}-u_{n-1}\right\|_{W^{\theta, p}(0, T ; D(A))} \\
& +k_{2}\|a\|_{L^{1}(-T, 0)}\left\|u_{n}-u_{n-1}\right\|_{C\left([0, T] ;(X, D(A))_{\theta+1-1 / p, p}\right)} .
\end{aligned}
$$


Summing both sides from $n=1$ to $m$ one gets

$$
\begin{aligned}
& \sum_{n=1}^{m}\left\|u_{n+1}-u_{n}\right\|_{C\left([0, T] ;(X, D(A))_{\theta+1-1 / p, p}\right)} \\
& \leq C_{T} k_{2}\|a\|_{L^{1}(-T, 0)}\left[(\theta p)^{-1 / p} C_{0}^{\prime}+1\right] \sum_{n=1}^{m}\left\|u_{n}-u_{n-1}\right\|_{W^{\theta, p}(0, T ; D(A))} \\
& +k_{2}\|a\|_{L^{1}(-T, 0)} \sum_{n=1}^{m}\left\|u_{n}-u_{n-1}\right\|_{C\left([0, T] ;(X, D(A))_{\theta+1-1 / p, p}\right)} .
\end{aligned}
$$

Substituting

$$
\begin{aligned}
& \sum_{n=1}^{m}\left\|u_{n}-u_{n-1}\right\|_{C\left([0, T] ;(X, D(A))_{\theta+1-1 / p, p}\right)} \\
& \leq \sum_{n=1}^{m}\left\|u_{n+1}-u_{n}\right\|_{C\left([0, T] ;(X, D(A))_{\theta+1-1 / p, p}\right)}+\left\|u_{1}-u_{0}\right\|_{C\left([0, T] ;(X, D(A))_{\theta+1-1 / p, p}\right)}
\end{aligned}
$$

in the last side of (3.35) one gets

$$
\begin{aligned}
& \sum_{n=1}^{m}\left\|u_{n+1}-u_{n}\right\|_{C\left([0, T] ;(X, D(A))_{\theta+1-1 / p, p}\right)} \\
& \leq C_{T} k_{2}\|a\|_{L^{1}(-T, 0)}\left[(\theta p)^{-1 / p} C_{0}^{\prime}+1\right] \sum_{n=1}^{m}\left\|u_{n}-u_{n-1}\right\|_{W^{\theta, p}(0, T ; D(A))} \\
& +k_{2}\|a\|_{L^{1}(-T, 0)} \sum_{n=1}^{m}\left\|u_{n+1}-u_{n}\right\|_{C\left([0, T] ;(X, D(A))_{\theta+1-1 / p, p}\right)} \\
& +k_{2}\|a\|_{L^{1}(-T, 0)}\left\|u_{1}-u_{0}\right\|_{C\left([0, T] ;(X, D(A))_{\theta+1-1 / p, p}\right)} .
\end{aligned}
$$

This implies

$$
\begin{aligned}
& \left(1-k_{2}\|a\|_{L^{1}(-T, 0)}\right) \sum_{n=1}^{m}\left\|u_{n+1}-u_{n}\right\|_{C\left([0, T] ;(X, D(A))_{\theta+1-1 / p, p}\right)} \\
& \leq C_{T} k_{2}\|a\|_{L^{1}(-T, 0)}\left[(\theta p)^{-1 / p} C_{0}^{\prime}+1\right] \sum_{n=1}^{m}\left\|u_{n}-u_{n-1}\right\|_{W^{\theta, p}(0, T ; D(A))} \\
& +k_{2}\|a\|_{L^{1}(-T, 0)}\left\|u_{1}-u_{0}\right\|_{C\left([0, T] ;(X, D(A))_{\theta+1-1 / p, p}\right)} .
\end{aligned}
$$

Letting $m \rightarrow \infty$ in (3.36) one obtains in view of (3.13)

$$
\begin{aligned}
& \left(1-k_{2}\|a\|_{L^{1}(-T, 0)}\right) \sum_{n=1}^{\infty}\left\|u_{n+1}-u_{n}\right\|_{C\left([0, T] ;(X, D(A))_{\theta+1-1 / p, p}\right)} \\
& \leq C_{T} k_{2}\|a\|_{L^{1}(-T, 0)}\left[(\theta p)^{-1 / p} C_{0}^{\prime}+1\right] \sum_{n=1}^{\infty}\left\|u_{n}-u_{n-1}\right\|_{W^{\theta, p}(0, T ; D(A))} \\
& +k_{2}\|a\|_{L^{1}(-T, 0)}\left\|u_{1}-u_{0}\right\|_{C\left([0, T] ;(X, D(A))_{\theta+1-1 / p, p}\right)}<\infty .
\end{aligned}
$$


Let $T$ satisfy $k_{2}\|a\|_{L^{1}(-T, 0)}<1$ besides $T \leq r$ and (3.12). Then by virtue of (3.37) and (3.13) one obtains

$$
\sum_{n=1}^{\infty}\left\|u_{n+1}-u_{n}\right\|_{C\left([0, T] ;(X, D(A))_{\theta+1-1 / p, p}\right)}<\infty .
$$

Hence $\left\{u_{n}\right\}$ is convergent in $C\left([0, T] ;(X, D(A))_{\theta+1-1 / p, p}\right)$. Since $u_{n} \rightarrow u$ in $W^{\theta, p}(0, T ; D(A))$, one concludes $u \in C\left([0, T] ;(X, D(A))_{\theta+1-1 / p, p}\right)$ and

$$
u_{n} \rightarrow u \text { in } W^{\theta, p}(0, T ; D(A)) \cap C\left([0, T] ;(X, D(A))_{\theta+1-1 / p, p}\right) .
$$

Let $T_{0}$ satisfy

$$
0<T_{0} \leq r, \quad C_{2}^{\prime} k_{2}\|a\|_{L^{1}\left(-T_{0}, 0\right)}\left[(\theta p)^{-1 / p} C_{0}^{\prime}+1\right]<1, \quad k_{2}\|a\|_{L^{1}\left(-T_{0}, 0\right)}<1 .
$$

Then, by the result just proved one has $u \in W^{\theta, p}(0, T ; D(A)) \cap$ $C\left(\left[0, T_{0}\right] ;(X, D(A))_{\theta+1-1 / p, p}\right)$. Hence

$$
\widehat{u} \in W^{\theta, p}(-r, T ; D(A)) \cap C\left(\left[-r, T_{0}\right] ;(X, D(A))_{\theta+1-1 / p, p}\right) .
$$

Suppose $T_{0}<T$. Then

$$
\left.\widehat{u}\right|_{\left[T_{0}-r, T_{0}\right]} \in W^{\theta, p}\left(T_{0}-r, T_{0} ; D(A)\right) \cap C\left(\left[T_{0}-r, T_{0}\right] ;(X, D(A))_{\theta+1-1 / p, p}\right),
$$

and $u$ satisfies

$$
\left\{\begin{array}{l}
\frac{d}{d t} u(t)=A u(t)+A_{1} u(t-r)+\int_{-r}^{0} a(s) A_{2} u(t+s) d s+f(t), T_{0} \leq t \leq T \\
u(s)=\left.\widehat{u}\right|_{\left[T_{0}-r, T_{0}\right]}(s), T_{0}-r \leq s \leq T_{0}
\end{array}\right.
$$

Therefore, by virtue of the result already proved

$$
u \in W^{\theta, p}(0, T ; D(A)) \cap C\left(\left[0, \min \left\{2 T_{0}, T\right\}\right] ;(X, D(A))_{\theta+1-1 / p, p}\right) .
$$

Continuing this process we can complete the proof of Theorem 5 .

Next, we consider the case where the following assumption is satisfied:

$$
\left\{\begin{array}{l}
\varphi_{1} \in W^{1+\theta, p}(-r, 0 ; D(A)) \cap C^{1}\left([-r, 0] ;(X, D(A))_{\theta+1-1 / p, p}\right), \\
f \in W^{\theta+1, p}(0, T ; X) \cap C\left([0, T] ;(X, D(A))_{\theta+1-1 / p, p}\right) \cap C^{1}\left([0, T] ;(X, D(A))_{\theta-1 / p, p}\right), \\
\varphi_{1}^{\prime}(0)=A \varphi_{1}(0)+A_{1} \varphi_{1}(-r)+\int_{-r}^{0} a(\sigma) A_{2} \varphi_{1}(\sigma) d \sigma+f(0) .
\end{array}\right.
$$

Theorem 6. If assumptions (II-2) and (II-4) are satisfied, then the solution u of (0.1) satisfies

$$
\begin{aligned}
& u \in W^{1+\theta, p}(0, T ; D(A)) \cap W^{2+\theta, p}(0, T ; X) \cap C^{1}\left([0, T] ;(X, D(A))_{\theta+1-1 / p, p}\right), \\
& u^{\prime \prime} \in C\left([0, T] ;(X, D(A))_{\theta-1 / p}\right) .
\end{aligned}
$$

Proof. If hypotheses (II-2) and (II-4) are satisfied, then (II-1) and (II-3) are also satisfied. In view of Theorem 2 and Theorem 5 it suffices to show

$$
u^{\prime} \in C\left([0, T] ;(X, D(A))_{\theta+1-1 / p}\right)
$$


Since (II-4) is satisfied, (II-3) is satisfied by $\varphi_{1}^{\prime}, f^{\prime}$ in place of $\varphi_{1}, f$. Therefore in view of Theorem 5 there exists a solution $v$ of the following problem

$$
\begin{aligned}
& \frac{d}{d t} v(t)=A v(t)+A_{1} v(t-r)+\int_{-r}^{0} a(s) A_{2} v(t+s) d s+f^{\prime}(t), \\
& v(s)=\varphi_{1}^{\prime}(s),-r \leq s \leq 0
\end{aligned}
$$

satisfying

$$
\begin{aligned}
& v \in W^{\theta, p}(0, T ; D(A)) \cap C\left([0, T] ;(X, D(A))_{\theta+1-1 / p, p}\right), \\
& d v / d t \in W^{\theta, p}(0, T ; X) \cap C\left([0, T] ;(X, D(A))_{\theta-1 / p}\right) .
\end{aligned}
$$

Since $u(t)=\varphi_{1}(0)+\int_{0}^{t} v(\tau) d \tau$ (c.f. Proof of Theorem 2),

$$
u^{\prime}=v \in C\left([0, T] ;(X, D(A))_{\theta+1-1 / p, p}\right) .
$$

\section{Solution Semigroup: Case $\theta>1 / p$}

In this section we assume that hypotheses (II-2) and (II-3) are satisfied. Let

$$
Z=W^{\theta, p}(-r, 0 ; D(A)) \cap C\left([-r, 0] ;(X, D(A))_{\theta+1-1 / p, p}\right) .
$$

For $\varphi_{1} \in Z$ let $u$ be the solution of $(0.1)$ with $f(t)=0$ :

$$
\begin{aligned}
& \frac{d}{d t} u(t)=A u(t)+A_{1} u(t-r)+\int_{-r}^{0} a(s) A_{2} u(t+s) d s, 0 \leq t<\infty, \\
& u(s)=\varphi_{1}(s), \quad-r \leq s \leq 0 .
\end{aligned}
$$

In view of Theorem $5 u$ satisfies

$$
\begin{aligned}
& u \in W^{\theta, p}(0, T ; D(A)) \cap C\left([0, T] ;(X, D(A))_{\theta+1-1 / p, p}\right), \\
& u^{\prime} \in W^{\theta, p}(0, T ; X) \cap C\left([0, T] ;(X, D(A))_{\theta-1 / p, p}\right) .
\end{aligned}
$$

Therefore $\widehat{u} \in W^{\theta, p}(-r, \infty ; D(A)) \cap C\left([-r, \infty) ;(X, D(A))_{\theta+1-1 / p, p}\right)$, where $u \in$ $W^{\theta, p}(-r, \infty ; D(A))$ means $u \in W^{\theta, p}(-r, T ; D(A)) \forall T>0$. This implies $\widehat{u}_{t} \in Z$ for $t \geq 0$. Therefore if we set

$$
S(t) \varphi_{1}=\widehat{u}_{t}, \quad t \geq 0
$$

$S(t)$ maps $Z$ to $Z$.

Let $\widehat{u}$ be the solution of (4.1), $v$ be the solution of (4.1) with the initial function $\widehat{u}_{\tau}$ and $w(t)=\widehat{u}(t+\tau)$ for $\tau>0, t>0$. Then

$$
\begin{aligned}
& \frac{d}{d t} v(t)=A v(t)+A_{1} v(t-r)+\int_{-r}^{0} a(s) v(t+s) d s, 0 \leq t<\infty, \\
& v(s)=\widehat{u}(\tau+s), \quad-r \leq s \leq 0, \\
& \frac{d}{d t} w(t)=\frac{d}{d t} \widehat{u}(t+\tau)=A \widehat{u}(t+\tau)+A_{1} \widehat{u}(t+\tau-r)+\int_{-r}^{0} a(s) \widehat{u}(t+\tau+s) d s \\
& =A w(t)+A_{1} w(t-r)+\int_{-r}^{0} a(s) w(t+s) d s, \quad 0 \leq t<\infty, \\
& w(s)=\widehat{u}(\tau+s),-r \leq s \leq 0 .
\end{aligned}
$$


Therefore $v=w$, and hence $v_{t}=w_{t}$. On the other hand

$$
\begin{aligned}
& v_{t}=S(t) \widehat{u}_{\tau}=S(t) S(\tau) \varphi_{1}, \\
& w_{t}(s)=w(t+s)=\widehat{u}(t+s+\tau)=\widehat{u}_{t+\tau}(s)=\left(S(t+\tau) \varphi_{1}\right)(s) .
\end{aligned}
$$

Thus

$$
S(t) S(\tau) \varphi_{1}=S(t+\tau) \varphi_{1}
$$

It is easy to see that the mapping $[0, T] \ni t \mapsto \widehat{u}_{t} \in C\left([-r, 0] ;(X, D(A))_{\theta+1-1 / p, p}\right)$ is continuous. The continuity of $[0, T] \ni t \mapsto \widehat{u}_{t} \in W^{\theta, p}(-r, 0 ; D(A))$ is shown in the following lemma.

Lemma 6. For $v \in W^{\theta, p}(-r, T ; D(A))$ the mapping $[0, T] \ni t \mapsto v_{t} \in W^{\theta, p}(-r, 0 ; D(A))$ is continuous.

Proof. The lemma is proved by the following step:

(i) For $w \in W^{1, p}(-r, T ; D(A)) \lim _{\tau \rightarrow t}\left\|w_{\tau}-w_{t}\right\|_{W^{\theta, p}(-r, 0 ; D(A))}=0$.

(ii) For $v \in W^{\theta, p}(-r, T ; D(A)), w \in W^{1, p}(-r, T ; D(A))$ such that $\|v-w\|_{W^{\theta, p}(-r, T ; D(A))}<\epsilon$ one has

$$
\begin{aligned}
& \left\|v_{\tau}-v_{t}\right\|_{W^{\theta, p}(-r, 0 ; D(A))} \\
& \leq\left\|w_{\tau}-w_{t}\right\|_{W^{\theta, p}(-r, 0 ; D(A))}+\left\|v_{\tau}-w_{\tau}\right\|_{W^{\theta, p}(-r, 0 ; D(A))}+\left\|v_{t}-w_{t}\right\|_{W^{\theta, p}(-r, 0 ; D(A))} \\
& \leq\left\|w_{\tau}-w_{t}\right\|_{W^{\theta, p}(-r, 0 ; D(A))}+2\|v-w\|_{W^{\theta, p}(-r, T ; D(A))}<\left\|w_{\tau}-w_{t}\right\|_{W^{\theta, p}(-r, 0 ; D(A))}+2 \epsilon .
\end{aligned}
$$

Hence the mapping $[0, T] \ni t \mapsto S(t) \varphi_{1}=\widehat{u}_{t} \in Z$ is continuous. Thus it has been shown that $\{S(t), t \geq 0\}$ is a $C_{0}$-semigroup.

The following result is an analog to Theorem 4.4 of E. Sinestrari [4]:

Theorem 7. The infinitesimal generator of $S(t)$ is given by

$$
\begin{aligned}
D(\Lambda)= & \left\{\varphi_{1} \in W^{1+\theta, p}(-r, 0 ; D(A)) \cap C^{1}\left([-r, 0] ;(X, D(A))_{\theta+1-1 / p, p}\right) ;\right. \\
& \left.\varphi_{1}^{\prime}(0)=A \varphi_{1}(0)+A_{1} \varphi_{1}(-r)+\int_{-r}^{0} a(\sigma) A_{2} \varphi_{1}(\sigma) d \sigma\right\} \\
\Lambda \varphi_{1}= & \varphi_{1}^{\prime} .
\end{aligned}
$$

Proof. We show

(i) $S(t) D(\Lambda) \subset D(\Lambda)$.

(ii) $D(\Lambda)$ is dense in $Z=W^{\theta, p}(-r, 0 ; D(A)) \cap C\left([-r, 0] ;(X, D(A))_{\theta+1-1 / p, p}\right)$.

(iii) $\Lambda \subset$ infinitesimal generator of $\{S(t)\}$.

(iv) $\Lambda: D(\Lambda) \subset W^{\theta, p}(-r, 0 ; D(A)) \rightarrow W^{\theta, p}(-r, 0 ; D(A))$ is closed.

(i) Let $\varphi_{1} \in D(\Lambda)$ and $u$ be the solution of (4.1). Then in view of Theorem 6 and its proof $u(0)=\varphi_{1}(0)$ and $u^{\prime}(0)=\varphi_{1}^{\prime}(0)$. Hence $\widehat{u} \in W^{1+\theta, p}(-r, \infty ; D(A)) \cap$ $C^{1}\left([-r, \infty] ;(X, D(A))_{\theta+1-1 / p, p}\right)$. Hence

$$
\widehat{u}_{t} \in W^{1+\theta, p}(-r, 0 ; D(A)) \cap C^{1}\left([-r, 0] ;(X, D(A))_{\theta+1-1 / p, p}\right) .
$$


For $t>0$

$$
\begin{aligned}
& \left(\widehat{u}_{t}\right)^{\prime}(0)=\lim _{s \rightarrow 0} \frac{\widehat{u}_{t}(s)-\widehat{u}_{t}(0)}{s}=\lim _{s \rightarrow-0} \frac{\widehat{u}(t+s)-\widehat{u}(t)}{s}=\lim _{s \rightarrow-0} \frac{u(t+s)-u(t)}{s} \\
& =u^{\prime}(t)=A u(t)+A_{1} \widehat{u}(t-r)+\int_{-r}^{0} a(s) A_{2} \widehat{u}(t+s) d s \\
& =A \widehat{u}_{t}(0)+A_{1} \widehat{u}_{t}(-r)+\int_{-r}^{0} a(s) A_{2} \widehat{u}_{t}(s) d s .
\end{aligned}
$$

Hence $\widehat{u}_{t} \in D(\Lambda)$.

(ii) Let $\varphi_{1} \in Z$ and $\widehat{u}_{t}=S(t) \varphi_{1}$. Set $\varphi_{\epsilon}=\int_{0}^{\epsilon} \widehat{u}_{t} d t \cdot \varphi_{\epsilon}(s)=\int_{0}^{\epsilon} \widehat{u}_{t}(s) d t=\int_{0}^{\epsilon} \widehat{u}(t+s) d t$.

$$
\begin{aligned}
& \varphi_{\epsilon}^{\prime}(s)=\frac{d}{d s}\left(\int_{0}^{\epsilon} \widehat{u}_{t} d t\right)(s)=\frac{d}{d s} \int_{0}^{\epsilon} \widehat{u}(t+s) d t=\int_{0}^{\epsilon} \widehat{u}^{\prime}(t+s) d t \\
& =\widehat{u}(\epsilon+s)-\widehat{u}(s)=\widehat{u}(\epsilon+s)-\varphi_{1}(s)=\widehat{u}_{\epsilon}(s)-\varphi_{1}(s),-r \leq s \leq 0 .
\end{aligned}
$$

Therefore

$$
\varphi_{\epsilon}^{\prime}=\widehat{u}_{\epsilon}-\varphi_{1} \in W^{\theta, p}(-r, 0 ; D(A)) \cap C\left([-r, 0] ;(X, D(A))_{\theta+1-1 / p, p}\right) .
$$

Hence

$$
\varphi_{\epsilon} \in W^{\theta+1, p}(-r, 0 ; D(A)) \cap C^{1}\left([-r, 0] ;(X, D(A))_{\theta+1-1 / p, p}\right) .
$$

By virtue of (4.2)

$$
\begin{aligned}
& A \varphi_{\epsilon}(0)+A_{1} \varphi_{\epsilon}(-r)+\int_{-r}^{0} a(\sigma) A_{2} \varphi_{\epsilon}(\sigma) d \sigma \\
& =A \int_{0}^{\epsilon} \widehat{u}(t) d t+A_{1} \int_{0}^{\epsilon} \widehat{u}(t-r) d t+\int_{-r}^{0} a(\sigma) A_{2} \int_{0}^{\epsilon} \widehat{u}(t+\sigma) d t d \sigma \\
& =\int_{0}^{\epsilon}\left(A \widehat{u}(t)+A_{1} \widehat{u}(t-r)+\int_{-r}^{0} a(\sigma) A_{2} \widehat{u}(t+\sigma) d \sigma\right) d t \\
& =\int_{0}^{\epsilon} \widehat{u}^{\prime}(t) d t=\widehat{u}(\epsilon)-\widehat{u}(0)=\widehat{u}_{\epsilon}(0)-\varphi_{1}(0)=\varphi_{\epsilon}^{\prime}(0) .
\end{aligned}
$$

Therefore $\varphi_{\epsilon} \in D(\Lambda)$. Since $[0, \infty) \ni t \mapsto \widehat{u}_{t} \in Z$ is continuous,

$$
\left\|\epsilon^{-1} \varphi_{\epsilon}-\varphi_{1}\right\|_{Z}=\left\|\frac{1}{\epsilon} \int_{0}^{\epsilon}\left(\widehat{u}_{t}-\varphi_{1}\right) d t\right\|_{Z} \leq \frac{1}{\epsilon} \int_{0}^{\epsilon}\left\|\widehat{u}_{t}-\varphi_{1}\right\|_{Z} d t \rightarrow 0 \text { as } \epsilon \rightarrow 0 .
$$

(iii) Let $\varphi_{1} \in D(\Lambda)$ and $u$ be the solution to (4.1). As was noted in the proof of (i) one has $\widehat{u} \in W^{1+\theta, p}(-r, \infty ; D(A)) \cap C^{1}\left([-r, \infty] ;(X, D(A))_{\theta+1-1 / p, p}\right)$. Hence

$$
\widehat{u}^{\prime} \in W^{\theta, p}(-r, \infty ; D(A)) \cap C\left([-r, \infty] ;(X, D(A))_{\theta+1-1 / p, p}\right) .
$$


Therefore

$$
\begin{aligned}
& \left\|\int_{0}^{1} \widehat{u}^{\prime}(t \sigma+\cdot) d \sigma-\widehat{u}^{\prime}\right\|_{W^{\theta, p}(-r, 0 ; D(A))}=\left\|\int_{0}^{1}\left(\widehat{u}^{\prime}(t \sigma+\cdot)-\widehat{u}^{\prime}\right) d \sigma\right\|_{W^{\theta, p}(-r, 0 ; D(A))} \\
& \leq \int_{0}^{1}\left\|\widehat{u}^{\prime}(t \sigma+\cdot)-\widehat{u}^{\prime}\right\|_{W^{\theta, p}(-r, 0 ; D(A))} d \sigma \rightarrow 0, \\
& \left\|\int_{0}^{1} \widehat{u}^{\prime}(t \sigma+\cdot) d \sigma-\widehat{u}^{\prime}\right\|_{C\left([-r, 0] ;(X, D(A))_{\theta+1-1 / p, p}\right)} \\
& \leq \int_{0}^{1}\left\|\widehat{u}^{\prime}(t \sigma+\cdot)-\widehat{u}^{\prime}\right\|_{C\left([-r, 0] ;(X, D(A))_{\theta+1-1 / p, p}\right)} d \sigma \rightarrow 0 .
\end{aligned}
$$

Hence

$$
\begin{aligned}
& \frac{S(t) \varphi_{1}-\varphi_{1}}{t}=\frac{\widehat{u}_{t}-\widehat{u}_{0}}{t}=\frac{\widehat{u}(t+\cdot)-\widehat{u}(\cdot)}{t}=\frac{1}{t} \int_{0}^{1} \frac{\partial}{\partial \sigma} \widehat{u}(t \sigma+\cdot) d \sigma \\
& =\int_{0}^{1} \widehat{u}^{\prime}(t \sigma+\cdot) d \sigma \rightarrow \widehat{u}^{\prime}(\cdot)=\Lambda \varphi_{1}
\end{aligned}
$$

in $Z$.

(iv) Let $\varphi_{1 n} \in D(\Lambda), \varphi_{1 n} \rightarrow \varphi_{1}, \varphi_{1 n}^{\prime}=\Lambda \varphi_{1 n} \rightarrow \psi$ in $Z$. Then $\varphi_{1}^{\prime}=\psi \in Z$. Hence

$$
\varphi_{1} \in W^{1+\theta, p}(-r, 0 ; D(A)) \cap C^{1}\left([-r, 0] ;(X, D(A))_{\theta+1-1 / p, p}\right) .
$$

Since $\varphi_{1 n} \rightarrow \varphi_{1}$ in $C\left([-r, 0] ;(X, D(A))_{\theta+1-1 / p, p}\right)$, one has $A \varphi_{1 n}(0) \rightarrow A \varphi_{1}(0)$, $A \varphi_{1 n}(-r) \rightarrow A \varphi_{1}(-r), \int_{-r}^{0} a(\sigma) A_{2} \varphi_{1 n}(\sigma) d \sigma \rightarrow \int_{-r}^{0} a(\sigma) A_{2} \varphi_{1}(\sigma) d \sigma$ in $(X, D(A))_{\theta-1 / p, p}$. One also has $\varphi_{1 n}^{\prime}(0) \rightarrow \psi(0)=\varphi_{1}^{\prime}(0)$ in $(X, D(A))_{\theta+1-1 / p, p}$. Therefore, from

$$
\varphi_{1 n}^{\prime}(0)=A \varphi_{1 n}(0)+A_{1} \varphi_{1 n}(-r)+\int_{-r}^{0} a(\sigma) A_{2} \varphi_{1 n}(\sigma) d \sigma
$$

it follows that

$$
\varphi_{1}^{\prime}(0)=A \varphi_{1}(0)+A_{1} \varphi_{1}(-r)+\int_{-r}^{0} a(\sigma) A_{2} \varphi_{1}(\sigma) d \sigma
$$

Therefore $\varphi_{1} \in D(\Lambda)$ and $\Lambda \varphi_{1}=\psi$.

\section{Remark on Hypothesis (II-2)}

Let $A$ be the realization in $L^{p}(\Omega), 1<p<\infty$, of a strongly elliptic linear partial differential operator of second order with the Dirichlet boundary condition, where $\Omega$ is a bounded domain in $\mathbb{R}^{n}$. Let $A_{i}, i=1,2$, be linear partial differential operators of second order in $\Omega$. Assume that the coefficients of $A, A_{i}, i=1,2$, and the boundary $\partial \Omega$ of $\Omega$ are sufficiently smooth. Then $D(A)=W^{2, p}(\Omega) \cap W_{0}^{1, p}(\Omega)$. Assume that $A$ has a bounded inverse. Suppose $1 / p<\theta<3 /(2 p)$. Then $0<2 \theta-2 / p<1 / p$. Hence by virtue of the results of R. Seeley [6] (also c.f. H. Triebel [7, Theorem 4.3.3]).

$$
\begin{aligned}
& \left(L^{p}(\Omega), D(A)\right)_{\theta-1 / p, p}=\left(L^{p}(\Omega), W^{2, p}(\Omega) \cap W_{0}^{1, p}(\Omega)\right)_{\theta-1 / p, p}= \\
& =B_{p, p}^{2 \theta-2 / p}(\Omega)=W^{2 \theta-2 / p, p}(\Omega) .
\end{aligned}
$$


Since

$$
A^{-1} \in \mathcal{L}\left(L^{p}(\Omega), W^{2, p}(\Omega) \cap W_{0}^{1, p}(\Omega)\right) \cap \mathcal{L}\left(W^{1, p}(\Omega), W^{3, p}(\Omega) \cap W_{0}^{1, p}(\Omega)\right),
$$

one has

$$
A^{-1} \in \mathcal{L}\left(W^{2 \theta-2 / p, p}(\Omega), W^{2+2 \theta-2 / p, p}(\Omega) \cap W_{0}^{1, p}(\Omega)\right) .
$$

Let $f \in\left(L^{p}(\Omega), D(A)\right)_{\theta-1 / p, p}$. In view of (5.1) and (5.2) $A^{-1} f \in W^{2+2 \theta-2 / p, p}(\Omega) \cap W_{0}^{1, p}(\Omega)$. Hence

$$
A_{i} A^{-1} f \in W^{2 \theta-2 / p, p}(\Omega)=\left(L^{p}(\Omega), D(A)\right)_{\theta-1 / p, p}, i=1,2 .
$$

Therefore

$$
A_{i} A^{-1} \in \mathcal{L}\left(\left(L^{p}(\Omega), D(A)\right)_{\theta-1 / p, p},\left(L^{p}(\Omega), D(A)\right)_{\theta-1 / p, p}\right), i=1,2 .
$$

Next, consider the case of the Neumann boundary condition. In this case

$$
D(A)=\left\{u \in W^{2, p}(\Omega) ; \partial u / \partial n=0 \text { on } \partial \Omega\right\},
$$

where $\partial u / \partial n$ is the outer conormal derivative with respect to $A$. Suppose $1 / p<\theta<3 /(2 p)+1 / 2, \theta \neq 1 / 2+1 / p$. Then, again by virtue of the results of R. Seeley [6] or H. Triebel [7]

$$
\left(L^{p}(\Omega), D(A)\right)_{\theta-1 / p, p}=B_{p, p}^{2(\theta-1 / p)}(\Omega)=W^{2(\theta-1 / p), p}(\Omega)
$$

and (5.3) follows as in the case of the Dirichlet boundary condition.

\section{References}

1. Di Blasio G., Lorenzi A. Identification Problems for Integro-Differential Delay Equations. Differential Integral Equations, 2003, vol. 16, no. 11, pp. 1385-1408.

2. Favini A., Tanabe H. Identification Problems for Integrodifferential Equations with Delay: an Improvement of the Results from G. Di Blasio and A. Lorenzi. Appear in Funkcialaj Ekvacioj.

3. Di Blasio G., Kunisch K., Sinestrari E. $L^{2}$-regularity for Parabolic Partial Integrodifferential Equations with Delay in the Highest-Order Derivatives. Journal of Mathematical Analysis and Applications, 1984, vol. 102, issue 1, pp. 38-57. DOI: 10.1016/0022-247X(84)90200-2

4. Sinestrari E. On a Class of Retarded Partial Differential Equations. Mathematische Zeitschrift, 1984, vol. 186, pp. 223-246.

5. Di Blasio G. Linear Parabolic Evolution Equations in $L^{p}$-Spaces. Annali di Matematica Pura ed Applicata (IV), 1984, vol. 138, issue 1, pp. 55-104. DOI: 10.1007/BF01762539

6. Seeley R. Interpolation in $L^{p}$ with Boundary Conditions. Studia Matematica, 1972, vol. 44, pp. $47-60$.

7. Triebel H. Interpolation Theory, Function Spaces, Differential Operators. Amsterdam, N.Y., Oxford, North-Holland, 1978.

Received November 28, 2016 


\section{РЕЗУЛЬТАТЫ РЕГУЛЯРНОСТИ И РАЗРЕШАЮЩИХ ПОЛУГРУПП ДЛЯ ФУНКЦИОНАЛЬНО- ДИФФЕРЕНЦИАЛЬНЫХ УРАВНЕНИЙ С ЗАПАЗДЫВАНИЕМ}

\section{А. Фавини, Х. Танабе}

Показано, что решения функционально-дифференциальных уравнений с запаздыванием в банаховом пространстве, существование и единственность которых показана раннее в работах А. Фавини и Х. Танабе, обладают дополнительными свойствами регулярности, если исходные данные и неоднородный член удовлетворяют некоторым предположениям о гладкости. Кроме того, получены некоторые результаты о разрешающих полугруппах.

Ключевые слова: функционально-дифференциальное уравнение с запаздыванием; регулярность решений; аналитической полугруппы; полугруппь решения; $C_{0}$ полугруппы; инфинитезимальный генератор.

Анджело Фавини, кафедра математики, Болонский университет (г. Болонья, Италия), favini@dm.unibo.it.

Хироки Танабе (г. Такарадзука, Япония), bacbx403@jttk.zaq.ne.jp.

Поступила в редакиию 28 ноября 2016 г. 